Article

\title{
A Compact UWB Bandpass Chip Filter on a GaAs Substrate with Modified Chebyshev Structure
}

\author{
Shanwen $\mathrm{Hu}{ }^{1, *} \mathbb{D}$, Yunqing $\mathrm{Hu}^{1}$, Yiting Gao ${ }^{1}$, Xiaodong Zhang ${ }^{2}$, Xinlei Zhang ${ }^{1}$, \\ Zixuan Wang ${ }^{1} \mathbb{D}$, Bo Zhou ${ }^{1}$, Zhikuang Cai ${ }^{1, *}$ and Yufeng Guo ${ }^{1}$ \\ 1 College of Electronic and Optical Engineering \& College of Microelectronics, \\ Nanjing University of Posts and Telecommunications, Nanjing 210023, China; \\ 1217022808@njupt.edu.cn (Y.H.); B17020603@njupt.edu.cn (Y.G.); 1219023504@njupt.edu.cn (X.Z.); \\ wangzixuan@njupt.edu.cn (Z.W.); nick.zhou@njupt.edu.cn (B.Z.); yfguo@njupt.edu.cn (Y.G.) \\ 2 School of Electronic \& Information Engineering, Suzhou University of Science and Technology, \\ Suzhou 215009, China; zhangxd_usts@126.com \\ * $\quad$ Correspondence: shanwenh@njupt.edu.cn (S.H.); whczk@njupt.edu.cn (Z.C.); Tel.: +86-025-85866131 (S.H.)
}

Received: 8 December 2019; Accepted: 8 February 2020; Published: 11 February 2020

check for updates

\begin{abstract}
Ultra-Wideband (UWB) systems are widely used in low-power, high-speed, high-security short-range wireless communication systems throughout digital homes and offices. In the RF front-end of a UWB system, bandpass filters (BPFs) are used to put through the passband signals and reject the stopband signals. Most UWB BPFs are designed with dielectric materials on circuit boards or LTCC technology. In this paper, a very compact fully integrated UWB chip filter is proposed and designed on a GaAs substrate with nitride as dielectric layers to meet the small size requirement of portable devices for next-generation UWB applications. The filter is constructed with a modified Chebyshev structure. The final filter circuit contains only four inductors instead of six for the conventional Chebyshev filter, which makes the chip more compact and cost effective. The filter is designed and fabricated on a $0.25 \mu \mathrm{m}$ GaAs pHEMT technology with a chip size of only $0.73 \mathrm{~mm} \times 0.51 \mathrm{~mm}$ including the chip edge and scribe line area, while the filter core area is only $0.61 \mathrm{~mm} \times 0.39 \mathrm{~mm}$, including bonding PADs. The measurement results illustrated that the proposed BPF shows a passband covering the frequency range of 3.1-9.0 GHz, the minimum passband insertion loss is only $1.5 \mathrm{~dB}$, the stopband rejection is better than $-30 \mathrm{~dB}$ throughout frequencies below $2 \mathrm{GHz}$ and above $12 \mathrm{GHz}$, S11 is less than $-16 \mathrm{~dB}$, and $\mathrm{S} 22$ is better than $-11 \mathrm{~dB}$ during the whole passband range. It demonstrated that the proposed filter can be considered as one of the most compact UWB filters.
\end{abstract}

Keywords: ultra-wideband; bandpass filter; on-chip elements; Chebyshev structure; GaAs pHEMT

\section{Introduction}

Ultra-Wideband (UWB) systems are widely used in short-distance wireless ranging, positioning and data transfer based on their features of low power, high speed, and high security. The bandwidth of a UWB standard system is $7.5 \mathrm{GHz}$ covering the 3.1 to $10.6 \mathrm{GHz}$ frequency range, while a data rate up to $500 \mathrm{Mb} / \mathrm{s}$ can be achieved. The signal power is extremely low as $-41 \mathrm{dBm}$ over the whole spectrum range (as shown in Figure 1); therefore, the UWB system is difficult to intercept, and interference on other wireless systems caused by UWB signals is also very low [1]. UWB techniques show large potential in next-generation communication scenarios, such as smart wearable devices, ranging and positioning for intelligent logistics and factories, and next-generation high-speed personal area networks (WPANs). Especially, UWB technology is commonly used in indoor positioning in order to present enhancements in terms of achieving high resolution and accuracy, low probability of interception, multipath immunity, and the ability to combine positioning and data communication 
in one system. Localization systems based on UWB technology achieve an accuracy of centimeters. In the UWB-based positioning system, interference with the ultra-wide spectrum may occur because of the misconfiguration. Interference may occur also due to the spread of the UWB signal over the bandwidths containing the frequency of the existing narrowband system [2]. Therefore, the UWB filter is a key component of indoor UWB positioning systems to remove interference and improve position accuracy. For these applications, the wireless circuit systems tend to be of high performance, low cost, and compact size to meet the requirements of next-generation portable and miniaturized devices. Then, the System in Package (SiP) solution is highly adopted due to its high integrated level; the passive and active circuit blocks are then realized as integrated circuit (IC) chips and mounted together with bonding wires in SiPs, including filters that are used to select the passband signal.

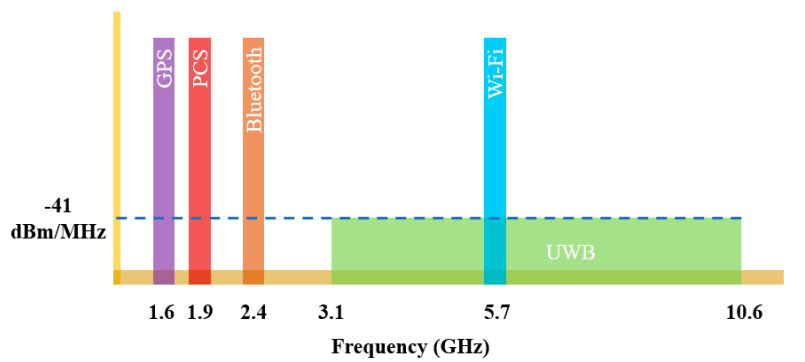

(a)

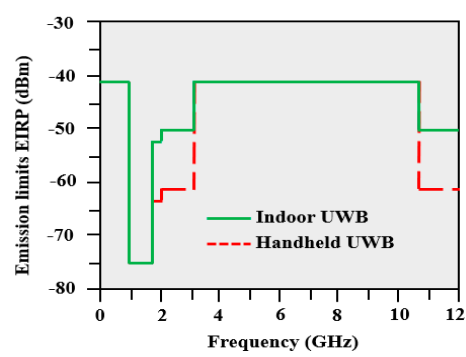

(b)

Figure 1. (a) The signal spectrum and (b) mask of an Ultra-Wideband (UWB) system.

The typical transmitter and receiver block diagrams are shown in Figure 2 [1]. At the transmitter end, a pulse signal is generated by the UWB pulse generator and then directly modulated with the input baseband data; a UWB bandpass filter is followingly used to put through the passband signal and reject the stopband signal before the signal is transmitted through the power amplifier and UWB antenna. At the receiver end, the signal is amplified to increase the amplitude range with a low-noise amplifier (LNA); then, another UWB bandpass filter is used to select the passband signal from the LNA, and the output baseband signal is finally received after the correction receiver.

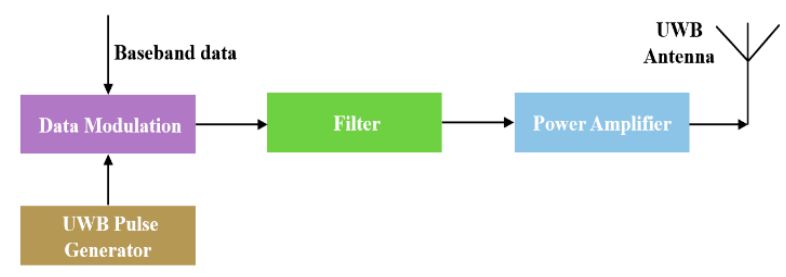

(a)

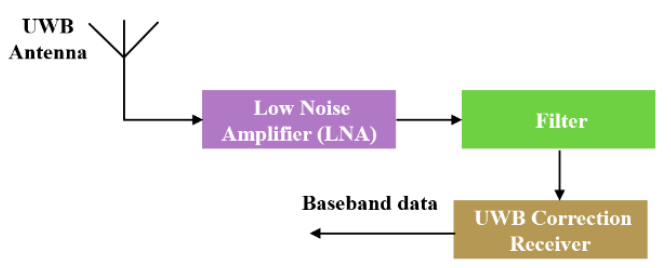

(b)

Figure 2. The typical block diagram of a UWB system (a) transmitter (b) receiver.

In this UWB circuit system, the bandpass filter is a key component and bottleneck for raising the level of integration and achieving high signal transfer performance. Most UWB bandpass filters (BPFs) are designed with dielectric materials on circuit boards or with LTCC technology. In the past decade, 
several bandpass filters are proposed for the UWB system [3-19]. The proposed filters have good performance of low loss, good matching, and high selectivity over the 3.1-10.6 GHz UWB frequency band. Unfortunately, these filters are off-chip components with a large size on a circuit board or in a package, which is difficult to be integrated in a UWB SiP system. Aiming to reduce the filter size, various advanced concepts have been proposed to designing a broadband filter based on the resonators, such as a stepped impedance resonator (SIR) [20], windmill resonator [21], or split ring resonator (SRR) [22]. Although these filters based on strip line resonators or ring-shape resonators show good performance on the circuit board level, they are still hard to be implemented at the on-chip level due to the limited quality factor of semiconductor materials. Most chip filters are still designed with lumped elements using on-chip inductors and capacitors. An on-chip bandpass filter is proposed in work [23] on a silicon integrated passive device (IPD) process. However, the passband is only 7.0-9.0 GHz. In work [24], a compact chip filter is proposed using standard silicon CMOS technology. The -3dB operation frequency band is 3.9-9.4 GHz. Silicon-based technology is cost effective and provides an ease of integration for UWB SiP solutions. However, the stopband rejection is not significant mainly due to the limited quality factor ( $Q$ factor) of the silicon substrate. A glass-based IPD technology is used in work [25] to realize an on-chip UWB filter using lumped elements with a high $Q$ factor due to its highly isolated glass substrate. The stopband rejection is good, but the passband frequency range is only 3.1-4.8 GHz. A glass-based on-chip filter using hybrid lumped/distributed elements is proposed in work [26], the passband frequency range is also around 3.1-4.8 GHz. The chip size of these two filters is relatively large: $5.0 \mathrm{~mm}^{2}$ and $11.5 \mathrm{~mm}^{2}$, respectively. Meanwhile, the fabrication cost is relatively high with the glass-based IPD technology. To overcome the low $Q$ properties of the silicon-based technology and high cost of the glass-based IPD technology, a GaAs substrate technology is a potential process to realize on-chip filters with balanced performance and cost properties [1]. In work [27], a 3.1-10.6 GHz on-chip UWB filter is proposed on a GaAs pHEMT process. The circuit size is $0.88 \mathrm{~mm} \times 0.88 \mathrm{~mm}$, while the passband insertion loss is less than $2.37 \mathrm{~dB}$. However, the stopband rejection is only $-10 \mathrm{~dB}$ near $2 \mathrm{GHz}$ and $12 \mathrm{GHz}$ frequencies. In this work, a novel compact chip filter is proposed to furtherly reduce the size of the UWB filter and improve the stopband rejection. Instead of modifying the structure of strip line resonators, ring resonators, inductors, or capacitors, as presented in previous works, we improve the performance of the proposed UWB filter in circuit topology by introducing a modified Chebyshev resonator network. Compared with the traditional six-order Chebyshev filter, the proposed filter is composed of only four resonate sections; it not only has the compact size advantage with the reduction of two inductors, but it also shows improved stopband rejection by generating two zero points at the adjacent frequency band. This filter is designed and fabricated on a $0.25 \mu \mathrm{m}$ GaAs pHEMT technology. The passband insertion loss of the proposed filter is only $1.5 \mathrm{~dB}$, a stopband rejection better than $-30 \mathrm{~dB}$ throughout frequencies below $2 \mathrm{GHz}$ and above $12 \mathrm{GHz}$ is realized, with a compact chip size of only $0.73 \mathrm{~mm} \times 0.51 \mathrm{~mm}$ including the chip edge and scribe line area. The filter core area size is only $0.61 \mathrm{~mm} \times 0.39 \mathrm{~mm}$ including bonding PADs. The material, structure, design theory, mechanism, simulations, and experiment demonstration of the proposed filter will be discussed in detail in the following sections.

\section{Materials and Methods}

\subsection{Materials and Technology}

The proposed filter is designed and fabricated using a commercial $0.25 \mu \mathrm{m}$ GaAs pHEMT technology, which is the mainstream technology to implement low-noise amplifiers, power amplifiers, and switches of RF front-ends for wireless communication systems. The passive devices based on this technology tend to have similar performance as they are designed on a GaAs IPD by the reason of the same GaAs substrate, nitride dielectric layers, and gold metal layers. A bandpass chip filter realized with this process has the potential to be easily integrated within the RF SiP systems. The simplified layer structure on a GaAs substrate with nitride dielectric layers is shown in Figure 1a. Only the 
passive dielectric layers and metal layers are used to implement this filter, so the active device layers are ignored in this design. There are two metal layers (M1 and M2), which are gold. The metal layers are insulated by several nitride dielectric layers and a polymide dielectric layer. The thickness of M1 is $1 \mu \mathrm{m}$, and M2 is a thick metal with an optional thickness $(2 \mu \mathrm{m}$ or $4 \mu \mathrm{m})$. A $4 \mu \mathrm{m}$ thickness is chosen for M2 in this design to enhance the power-handling capacity of inductors and reduce the metal loss of metal lines, inductors, and conductors. Layers M1 and M2 are connected by the metallization backside via holes. The second nitride layer is adopted to form the MIM capacitor. The inductor is realized by the spiral structure composed of M1 and M2.

The 3D structure of the proposed UWB chip filter is shown in Figure 3b. The signal is fed in and out of the filter through input/output GSG pads. The transmission metal lines are designed to achieve good transmission matching using the M1 or M2 metal layer. The spiral inductors are designed as a square or rectangular shape to generate a miniaturized chip area; both M1 and M2 metal layers are used for inductors to improve the power-handling capacity and reduce metal loss. The plate capacitors are used in this chip filter, which is composed of bottom metal M1, top metal M2, and nitride dielectric layer. Several LC resonator networks are constructed by these serial or shunt-connected inductors and capacitors to generate multiple resonate poles and zeros to cover the wide passband range and realize stopband rejection for this broadband UWB filter. The circuit model and filter design mechanism will be discussed and demonstrated in detail in the following sections.

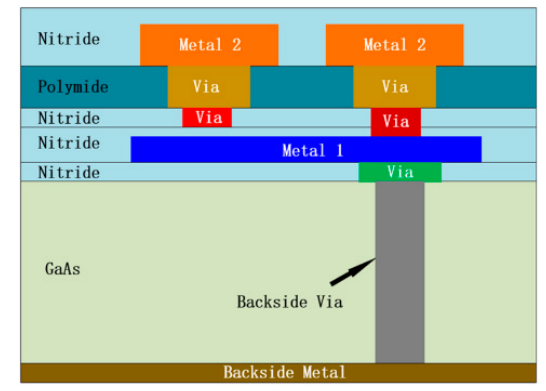

(a)

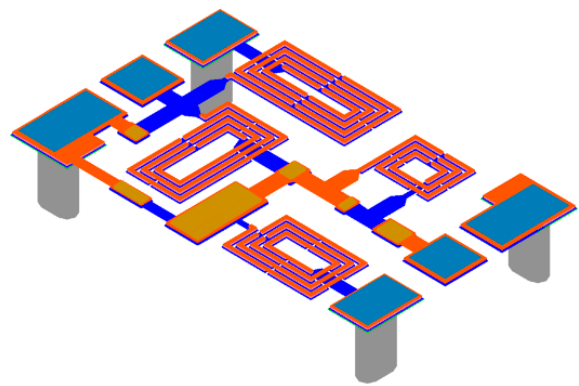

(b)

Figure 3. (a) Simplified layer structure of GaAs pHEMT without active transistor (b) 3D structure of the proposed UWB chip filter.

\subsection{Filter Structure}

Chebyshev filters are traditionally used for LC resonator-type filters due to its features of a flat passband response, good stopband, rejection, and simple structure. A sixth-order Chebyshev filter is shown in Figure 4. This traditional Chebyshev filter is composed of six LC resonator sections. The odder resonators 1,3,5 are constructed by shunt LC networks, and the even resonators 2,4,6 are constructed by series LC networks. These shunt and series LC resonators are serially connected to form a six-order filter structure, which can be used to implement the broadband UWB filter.

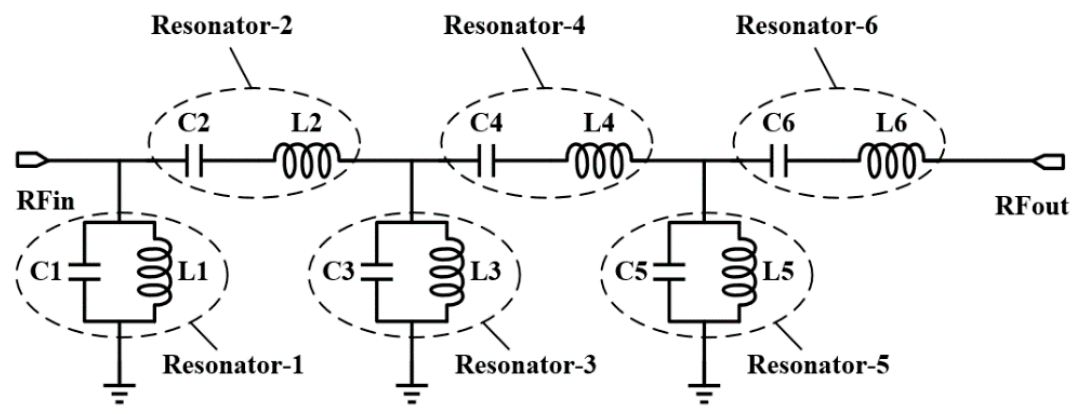

Figure 4. Circuit model of a sixth-order Chebyshev filter. 
The resonate frequency of each section can be simply written as the following equations:

$$
\begin{array}{lll}
f_{p 1}=\frac{1}{2 \pi \sqrt{L_{1} C_{1}}} & f_{p 2}=\frac{1}{2 \pi \sqrt{L_{2} C_{2}}} \quad f_{p 3}=\frac{1}{2 \pi \sqrt{L_{3} C_{3}}} . \\
f_{p 4}=\frac{1}{2 \pi \sqrt{L_{4} C_{4}}} \quad f_{p 5}=\frac{1}{2 \pi \sqrt{L_{5} C_{5}}} \quad f_{p 6}=\frac{1}{2 \pi \sqrt{L_{6} C_{6}}} .
\end{array}
$$

Six different resonate frequencies can be derived based on this traditional sixth-order Chebyshev filter; then, a wide passband frequency range is realized for UWB filters. However, the stopband rejection is weak with this traditional structure, since no zero resonator is utilized in this structure. On the other hand, this structure is not suitable for compact filter design, since there are in total 12 lumped elements including six inductors and six capacitors. A large area will be occupied with such a number of lumped elements on a single semiconductor GaAs chip, especially for the spiral inductors, whose size can be illustrated in Figure $3 \mathrm{~b}$.

A modified Chebyshev filter is proposed in this work to improve the stopband rejection and reduce the size of the UWB filter. The 2D layout topology of this filter is shown in Figure 5a, and the circuit model is shown in Figure 5b. The proposed filter is composed of four LC resonator sections. Resonator-1 and resonator- 2 are a shunt LC network and series LC network, respectively, which is the same as that in the traditional Chebyshev filter to ensure two poles for the passband response. Two modified LC resonator network resonators-3 and 4-are introduced in the proposed filter. Both of them are composed of a shunt LC network in serial with a capacitor. The total number of lumped elements in this novel filter is 10, including four inductors and six capacitors, as shown in Figure 5, which is two elements less compared with the traditional Chebyshev filter in Figure 4. Especially, two large inductors L5 and L6 in Figure 4 are removed in this novel filter. Consequently, this modified Chebyshev filter is much more compact compared with the traditional Chebyshev filter; therefore, it is more suitable to realize the small on-chip filter for UWB systems. On the other hand, the proposed filter shows improved stopband rejection properties since two additional zeros are introduced by the modified resonator-3 and resonator-4, which will be analyzed in detail in the following sections.

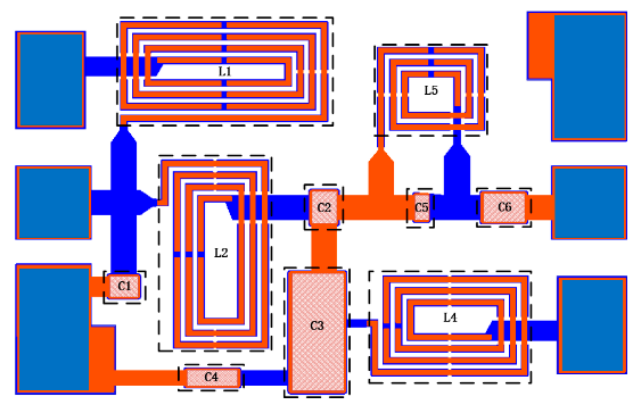

(a)

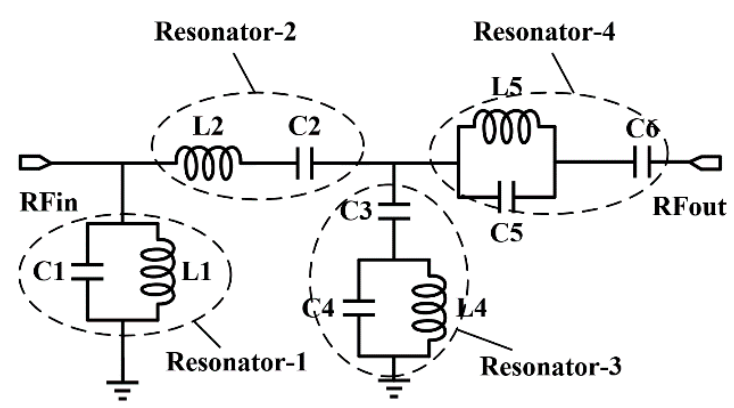

(b)

Figure 5. (a) 2D topology and (b) circuit model of the proposed filter.

\subsection{Filter Model}

The circuit model of the proposed filter can be simplified as a Z-model circuit as illustrated in Figure 6. The impedance $Z_{1}, Z_{2}, Z_{3}$, and $Z_{4}$ of each resonator section can be written as the following equations.

$$
\begin{aligned}
& Z_{1}=\frac{j \omega L_{1} \times \frac{1}{j \omega C_{1}}}{j \omega L_{1}+\frac{1}{j \omega C_{1}}}=\frac{j \omega L_{1}}{1-\omega^{2} L_{1} C_{1}} \\
& Z_{2}=j \omega L_{2}+\frac{1}{j \omega C_{2}}=\frac{1-\omega^{2} L_{2} C_{2}}{j \omega C_{2}}
\end{aligned}
$$




$$
\begin{aligned}
& Z_{3}=\frac{j \omega L_{4} \times \frac{1}{j \omega C_{4}}}{j \omega L_{4}+\frac{1}{j \omega C_{4}}}+\frac{1}{j \omega C_{3}}=\frac{1-\omega^{2} L_{4} C_{4}-\omega^{2} L_{4} C_{3}}{j \omega C_{3}\left(1-\omega^{2} L_{4} C_{4}\right)} \\
& Z_{4}=\frac{j \omega L_{5} \times \frac{1}{j \omega C_{5}}}{j \omega L_{5}+\frac{1}{j \omega C_{5}}}+\frac{1}{j \omega C_{6}}=\frac{1-\omega^{2} L_{5} C_{5}-\omega^{2} L_{5} C_{6}}{j \omega C_{6}\left(1-\omega^{2} L_{5} C_{5}\right)}
\end{aligned}
$$

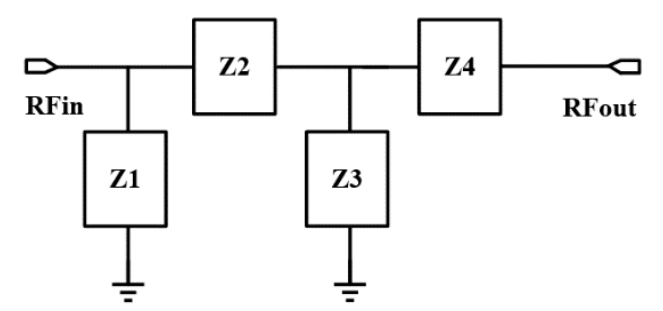

Figure 6. Simplified Z-model of the proposed filter.

The resonate poles of this circuit can be derived by assuming the shunt impedance to be infinite $Z_{1} \& Z_{3}=\infty$, and the serial impedance to be zero $Z_{2} \& Z_{4}=0$. The zeros can be derived by assuming the shunt impedance to be zero $Z_{3}=0$ and the serial impedance to be infinite $Z_{4}=\infty$. Therefore, the pole and zero frequency points of the proposed filter can be written as following equations after mathematic calculation.

$$
\begin{gathered}
f_{p 1}=\frac{1}{2 \pi \sqrt{L_{1} C_{1}}} \quad f_{p 2}=\frac{1}{2 \pi \sqrt{L_{2} C_{2}}} \quad f_{p 3}=\frac{1}{2 \pi \sqrt{L_{4} C_{4}}} \quad f_{p 4}=\frac{1}{2 \pi \sqrt{L_{5} C_{5}+L_{5} C_{6}}} \\
f_{z 1}=\frac{1}{2 \pi \sqrt{L_{4} C_{4}+L_{4} C_{3}}} \quad f_{z 2}=\frac{1}{2 \pi \sqrt{L_{5} C_{5}}}
\end{gathered}
$$

As it is shown in Equation (6), four resonate poles are generated by the proposed filter, which is two poles less compared with the traditional sixth-order Chebyshev filter. However, it is enough to design a broadband UWB filter with these four poles to achieve the desired passband flatness and wide frequency range, which will be demonstrated by following the simulation and experiment results. Two additional zeros are introduced by the proposed filter. The zero points will be generated at lower stopband frequency and higher stopband frequency, respectively. Therefore, the stopband rejection at both lower and higher frequencies will be improved by this proposed filter.

\subsection{Filter Design and Simulation}

The traditional Chebyshev filter can be designed based on the classical Chebyshev equations, and the value of the circuit elements can be easily generated using a filter design software. The values of inductors and capacitors of the sixth-order Chebyshev filter shown in Figure 4 are designed as shown in Table 1. The filter is initially designed with ideal lossless passive elements without considering the material loss and other parasitic effects for easier analysis and faster design. The proposed modified Chebyshev filter is also designed and optimized to achieve good passband and stopband filter performance for UWB systems. The ideal lossless elements' values are shown in Table 2. Compared with the traditional filter, two large inductors are removed, which will reduce the size of the filter chip. It is shown in Table 2 that $\mathrm{C} 3$ is larger in the modified Chebyshev filter. However, this capacitor area is still relatively small compared with the on-chip inductors, which is illustrated in Figure 5.

In Table 2, L1 and C1 of resonator- 1 is $2.20 \mathrm{nH}$ and $0.35 \mathrm{pF}$ respectively, while $\mathrm{L} 2$ and C2 of resonator-2 is $1.60 \mathrm{nH}$ and $0.49 \mathrm{pF}$ respectively, which are similar values compared with those of the traditional filter in Table.1. Therefore, resonator- 1 and resonator- 2 in the proposed filter are designed similarly to the traditional Chebyshev filter to ensure two poles in the passband. For resonator-3, $\mathrm{C} 3=3.87 \mathrm{pF}, \mathrm{C} 4=0.48 \mathrm{pF}$, and $\mathrm{L} 4=1.50 \mathrm{nH}$. If we are putting these values into Equation (7), then a 
zero-point frequency $f_{\mathrm{z} 1}$ can be designed as $1.97 \mathrm{GHz}$. For resonator- $4, \mathrm{C} 5=0.20 \mathrm{pF}, \mathrm{C} 6=0.72 \mathrm{pF}$, and $\mathrm{L} 5=0.60 \mathrm{nH}$. If putting these values into Equation (7), then another zero-point frequency $f_{\mathrm{z} 2} \mathrm{can}$ be designed as $14.53 \mathrm{GHz}$. Consequently, two zeros at lower and higher stopband frequencies are realized respectively for the proposed filter. Therefore, both the passband and stopband characteristics of the filter are well designed and optimized base on the proposed structure and design mechanism.

Table 1. The ideal lossless elements values of the traditional Chebyshev filter.

\begin{tabular}{cccc}
\hline Lumped Elements & Value & Lumped Elements & Value \\
\hline L1 & $2.38 \mathrm{nH}$ & $\mathrm{C} 1$ & $0.29 \mathrm{pF}$ \\
L2 & $1.58 \mathrm{nH}$ & $\mathrm{C} 2$ & $0.45 \mathrm{pF}$ \\
L3 & $1.10 \mathrm{nH}$ & $\mathrm{C} 3$ & $0.63 \mathrm{pF}$ \\
L4 & $1.75 \mathrm{nH}$ & $\mathrm{C} 4$ & $0.40 \mathrm{pF}$ \\
L5 & $1.24 \mathrm{nH}$ & $\mathrm{C} 5$ & $0.56 \mathrm{pF}$ \\
L6 & $0.81 \mathrm{nH}$ & $\mathrm{C} 6$ & $0.86 \mathrm{pF}$ \\
\hline
\end{tabular}

Table 2. The ideal lossless elements values of the proposed modified Chebyshev filter.

\begin{tabular}{cccc}
\hline Lumped Elements & Value & Lumped Elements & Value \\
\hline L1 & $2.20 \mathrm{nH}$ & $\mathrm{C} 1$ & $0.35 \mathrm{pF}$ \\
L2 & $1.60 \mathrm{nH}$ & $\mathrm{C} 2$ & $0.49 \mathrm{pF}$ \\
L4 & $1.50 \mathrm{nH}$ & $\mathrm{C} 3$ & $3.87 \mathrm{pF}$ \\
L5 & $0.60 \mathrm{nH}$ & $\mathrm{C} 4$ & $0.48 \mathrm{pF}$ \\
& & $\mathrm{C} 5$ & $0.20 \mathrm{pF}$ \\
& & $\mathrm{C} 6$ & $0.72 \mathrm{pF}$ \\
\hline
\end{tabular}

The S21 response of the proposed and traditional filter are both simulated as shown in Figure 7. The passband frequency range is from 3 to $11 \mathrm{GHz}$, the passband insertion loss is almost $0 \mathrm{~dB}$, since all of the inductors and capacitors used are ideal lumped elements. Figure 7 shows that a zero-frequency point of the proposed filter is introduced at a $2 \mathrm{GHz}$ low stopband frequency point, and another zero point is introduced at a $14.5 \mathrm{GHz}$ high-frequency point. These simulated zero points matched very well with the calculated results from Equation (7), which are $1.97 \mathrm{GHz}$ and $14.53 \mathrm{GHz}$, respectively. The rejection of the proposed filter at $2 \mathrm{GHz}$ is $-51.4 \mathrm{~dB}$, while that of the traditional filter is only $-39.2 \mathrm{~dB}$, which shows a $-12.2 \mathrm{~dB}$ improvement of rejection at $2 \mathrm{GHz}$ with this novel UWB filter. The rejection of the proposed filter at $14.5 \mathrm{GHz}$ is $-54.7 \mathrm{~dB}$, while that of the traditional filter is only $-20.5 \mathrm{~dB}$, which shows a $-34.2 \mathrm{~dB}$ improvement of rejection at $14.5 \mathrm{GHz}$ with this novel UWB filter. It demonstrated that the proposed filter not only has the compact size advantage with the reduction of two inductors, but also shows improved stopband rejection at the adjacent frequency band. The trade-off sacrifice of this proposed filter is that it shows weaker rejection at the frequencies lower than $1.9 \mathrm{GHz}$. These frequencies are relatively far from the UWB operation band, so that the interference shows less effect on the UWB system. On the other hand, the rejection at these frequencies are all lower than $-36 \mathrm{~dB}$, which means the rejection is good enough to satisfy UWB filtering applications.

To further demonstrate the advantage and performance of the proposed filter, it is designed on a 0.25 um GaAs pHEMT process. The 3D and top-view layout structures are illustrated in Figures $3 \mathrm{~b}$ and $5 \mathrm{a}$. The inductors in the circuit model are designed as lumped spiral inductors in the layout. The capacitors are designed as plate capacitors, and they are connected by metal layers M1 and M2; the dielectric for capacitors is the nitride layer. An EM simulation is performed to relatively accurately predict the transfer performance of the proposed filter by calculating the parasitic effect of the material and devices. The simulated $S$ parameters of the proposed filter are shown in Figure 8. The passband insertion loss $\mathrm{S} 21$ of the proposed filter is about $1.5 \mathrm{~dB}$, which was mainly introduced by the substrate loss and metal parasitic resistance. The frequency range of the passband is from $3.1 \mathrm{GHz}$ to $9.0 \mathrm{GHz}$, which is $2 \mathrm{GHz}$ less compared with that of the filter using ideal elements. The reason of the high-frequency S21 degradation is mainly caused by the GaAs process we adopted, which has a cut-off 
frequency of only $29.5 \mathrm{GHz}$. Therefore, the inductors and capacitors on this GaAs substrate show strong self-resonance behavior when operation frequencies exceed $10 \mathrm{GHz}$. However, the $3.1-9.0 \mathrm{GHz}$ passband covering range is sufficient in real UWB application, since the bandwidth of the UWB signal pulse is normally covered in this range. A zero point at $1.75 \mathrm{GHz}$ with $-42 \mathrm{~dB}$ rejection is introduced as shown in Figure 8, and another zero point is at $13 \mathrm{GHz}$ with $-42 \mathrm{~dB}$ rejection. The return loss $\mathrm{S} 11$ is lower than $-18 \mathrm{~dB}$, and S22 is lower than $-10 \mathrm{~dB}$ during the whole operation frequency.

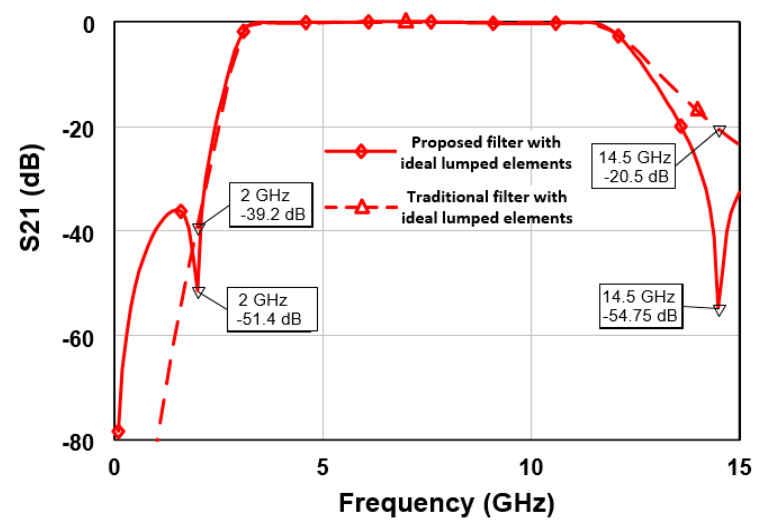

Figure 7. Simulated S21 response of the proposed and traditional filter with ideal elements.

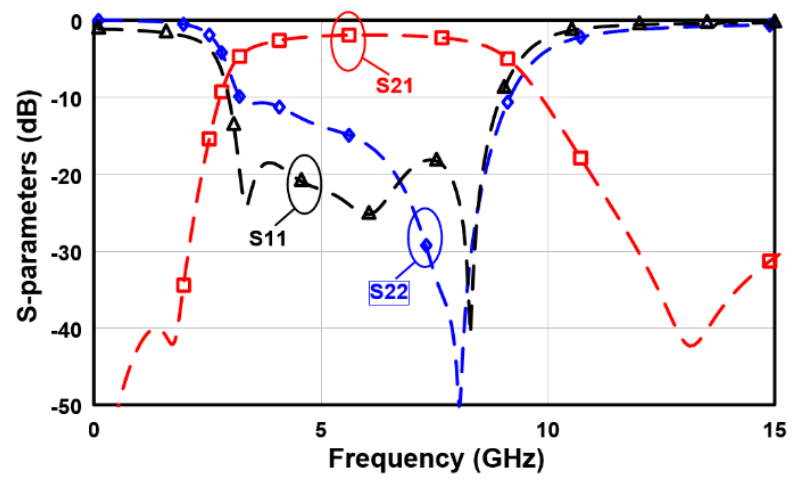

Figure 8. EM-simulated S parameters of the proposed filter on GaAs substrate.

The current distributions of the proposed filter at frequencies of $2 \mathrm{GHz}, 4 \mathrm{GHz}, 6 \mathrm{GHz}, 8 \mathrm{GHz}$, and $12 \mathrm{GHz}$ are simulated and illustrated in Figure 9. The normalized current density is $200 \mathrm{~A} / \mathrm{m}$. It shows that at the $4 \mathrm{GHz}, 6 \mathrm{GHz}$ and $8 \mathrm{GHz}$ passband frequencies, the current is well transferred to the output. At the $2 \mathrm{GHz}$ and $12 \mathrm{GHz}$ stopband frequencies, the current is constrained in the zero resonators and reflected back to the input, so that the signal transfer through this filter is rejected at these frequencies. Therefore, the proposed filter shows the capacity of letting the passband signals go through the device and rejecting the stopband signals. Consequently, a compact chip filter for UWB systems with low loss and high selectivity is well designed and optimized. 


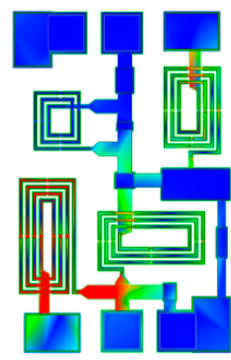

$2 \mathrm{GHz}$

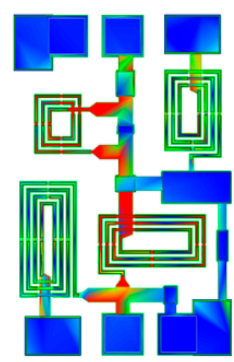

$4 \mathrm{GHz}$

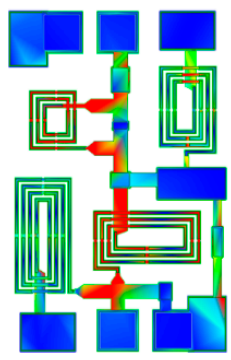

$6 \mathrm{GHz}$

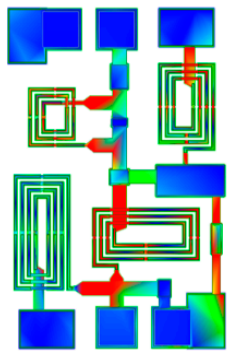

8GHz

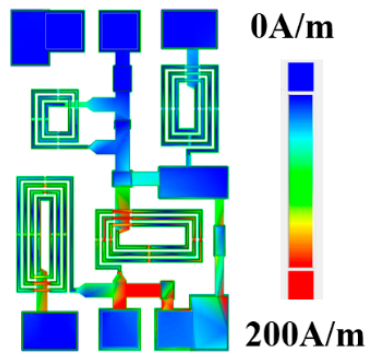

$12 \mathrm{GHz}$

Figure 9. Current distribution of the proposed filter at different frequencies.

\section{Results}

The proposed filter is finally fabricated on a 0.25 um GaAs pHEMT process; the chip photo is shown in Figure 10. All the six capacitors are small with little chip area consumption, and there are only four inductors remained in the proposed filter to furtherly reduce the chip size. Finally, a very compact filter chip size of $0.73 \mathrm{~mm} \times 0.51 \mathrm{~mm}$ is achieved including chip edge and scribe line area, and the filter core area is only $0.61 \mathrm{~mm} \times 0.39 \mathrm{~mm}$ including bonding PADs.

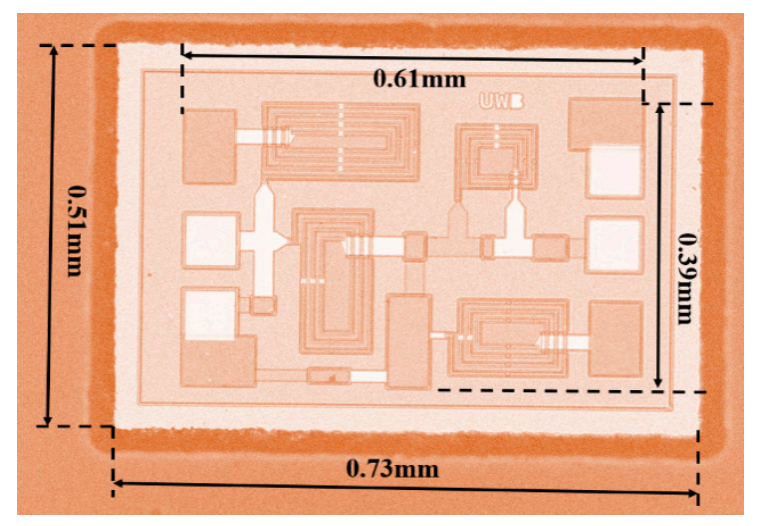

Figure 10. Chip photo of the proposed filter.

The proposed UWB chip filter is measured on an RF probe station to avoid the parasitic effects introduced by the packaging, circuit board, or connection wires. $S$ parameters are measured for the proposed filter, whose amplitudes are compared with the simulation results as shown in Figure 11. The measured insertion loss S21 is well matched with the simulated results, the minimum insertion loss in the passband is only $1.5 \mathrm{~dB}$, and the $3-\mathrm{dB}$ passband bandwidth is from 3.1 to $9.0 \mathrm{GHz}$. For the stopband performance, two zeros are realized at the $1.75 \mathrm{GHz}$ and $13.5 \mathrm{GHz}$ frequency points, while the rejection at $2 \mathrm{GHz}$ is up to $-34 \mathrm{~dB}$, and it is $-33 \mathrm{~dB}$ at $12 \mathrm{GHz}$. Two matching frequency points of S11 match well with the simulated results, and the measured S11 is lower than $-16 \mathrm{~dB}$ during the whole passband. The matching frequency point of $\mathrm{S} 22$ shifts about $2 \mathrm{GHz}$ to the lower frequency end compared with the simulated results, which is mainly because of the parasitic effects and inter-device coupling of the shunt-series LC network of resonator-3 and resonator-4 at high frequencies. The measured S22 is lower than $-11 \mathrm{~dB}$ during the whole passband. 


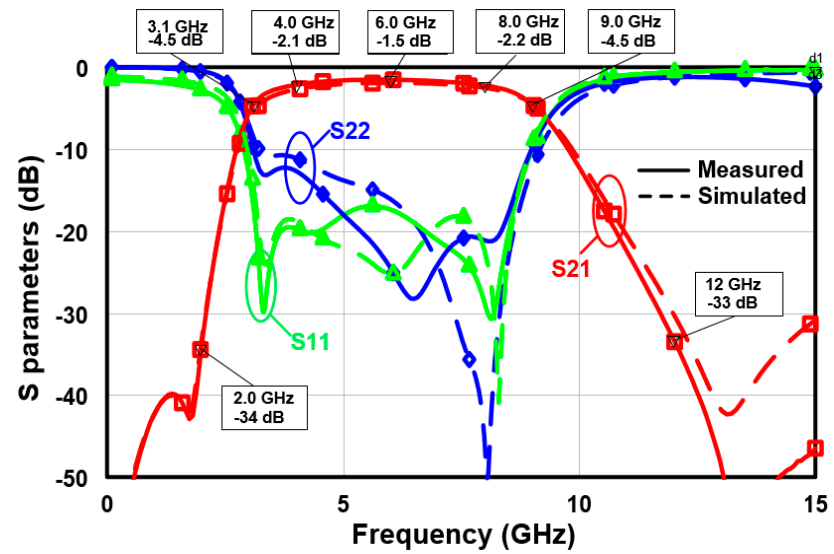

Figure 11. Measured and simulated S parameters of the proposed filter.

The phase response of S21 is measured for the proposed filter, which is compared with the simulation result as shown in Figure 12. It shows that the phase response of S21 changes linearly during the whole 2.9-9.0 GHz frequency band, indicating the small phase distortion of the proposed filter. Group delay is usually used to judge the nonlinear distortion of a filter. If a group delay ripple is large during the passband, then the signal transmit time through the device shows nonlinear variation at different frequencies. The measured and simulated group delay of the proposed filter are compared in Figure 13. The maximum passband group delay is located at $3.0 \mathrm{GHz}$, which is $0.39 \mathrm{~ns}$. The minimum passband group delay is $0.12 \mathrm{~ns}$ at $6 \mathrm{GHz}$. Therefore, the group delay variation during the whole passband is only $0.27 \mathrm{~ns}$, which is extremely low for such a wideband chip filter.

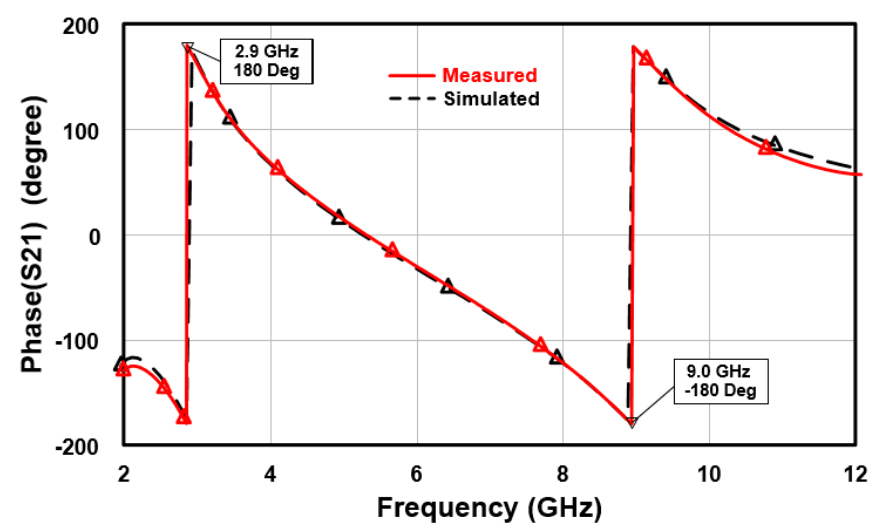

Figure 12. Measured and simulated phase response of the proposed filter.

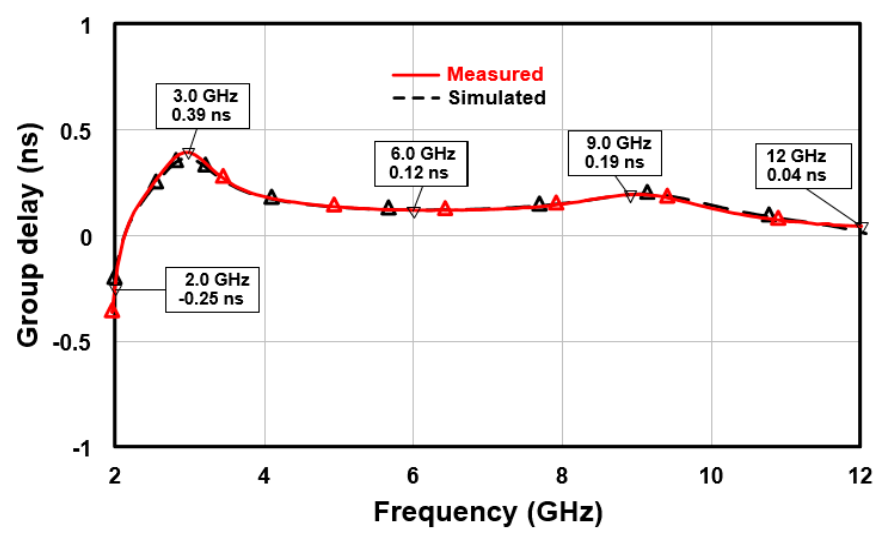

Figure 13. Measured and simulated group delay of the proposed filter. 


\section{Discussion}

Research on UWB bandpass filters for next-generation portable UWB systems mainly aims for their size reduction, low loss in the passband, and high rejection in the stopband. In this work, a modified Chebyshev structure is proposed to reduce the number of inductors to reduce the filter size. On the other hand, two additional transmission zeros are introduced by this modified structure to improve rejection in the stopband. The low passband loss is also achieved by an optimized design on the 0.25 um pHEMT substrate. Finally, the bandpass chip filter was fabricated to confirm, by means of the measurement results, the theory and design methods proposed in this work.

Many studies have been focused on improving the performance of the UWB chip filter in recent years. Performances of several reported UWB chip filters based on silicon, glass IPD, and GaAs technology are summarized and compared in Table 3. The passband of the chip filter reported in work [23] is only 7.0-9.0 GHz. In work [24], a compact chip filter is reported using standard silicon CMOS technology, and the passband is 3.9-9.4 GHz. However, the rejection at $2 \mathrm{GHz}$ and $12 \mathrm{GHz}$ is only $-15 \mathrm{~dB}$. The glass-based IPD chip filters in work [25] and [26] are designed only for 3.1-4.8 GHz narrowband UWB systems, and the chip size is too large. In work [27], a very compact 3.1-10.6 GHz GaAs-based IPD chip filter is successfully designed with low passband loss and good return loss. However, the stopband rejection is only $-10 \mathrm{~dB}$ near the $2 \mathrm{GHz}$ and $12 \mathrm{GHz}$ frequencies. For the design proposed in this work, the chip size is only $0.61 \mathrm{~mm} \times 0.39 \mathrm{~mm}$, the minimum passband loss is low as $1.5 \mathrm{~dB}$, and the maximum return loss is $-11 \mathrm{~dB}$. Moreover, the rejection is up to $-34 \mathrm{~dB}$ at $2 \mathrm{GHz}$ and $-33 \mathrm{~dB}$ at $12 \mathrm{GHz}$.

Table 3. Comparison of chip filters for UWB applications. IPD: integrated passive device.

\begin{tabular}{cccccc}
\hline Ref. & $\begin{array}{c}\text { Passband } \\
\text { (GHz) }\end{array}$ & $\begin{array}{c}\text { Insertion Loss } \\
\text { (dB) }\end{array}$ & $\begin{array}{c}\text { Return Loss } \\
(\mathbf{d B})\end{array}$ & $\begin{array}{c}\text { Chip Size } \\
(\mathbf{m m} \times \mathbf{m m})\end{array}$ & Technology \\
\hline$[23]$ & $7.0-9.0$ & 2.5 & -12 & $1.6 \times 1.2$ & Silicon IPD \\
{$[24]$} & $3.9-9.4$ & 1.89 & -11 & $0.54 \times 0.497$ & Silicon CMOS \\
{$[25]$} & $3.1-4.8$ & 2.5 & -8 & 3.8 & Glass IPD \\
{$[26]$} & $3.1-4.8$ & 2.4 & -12 & 5.0 & Glass IPD \\
{$[27]$} & $3.1-10.6$ & 1.0 & -15 & $0.88 \times 0.88$ & GaAs pHEMT \\
This work & $3.1-9.0$ & 1.5 & -11 & $0.61 \times 0.39$ & GaAs pHEMT \\
\hline
\end{tabular}

\section{Conclusions}

A compact UWB chip filter with a modified Chebyshev structure is designed and proposed in this work. Two traditionally connected LC resonators are adopted to generate two poles to ensure passband properties. Another two shunt LC resonators in serial with a capacitor are adopted to generate the other two poles for passband, and two additional zeros at $2 \mathrm{GHz}$ and $14.5 \mathrm{GHz}$ are also introduced by these two resonators, which can improve the stopband rejection. The structure of the proposed filter is analyzed, and the parameters of the equivalent circuit are calculated and discussed. The inductors and capacitors are optimized to achieve optimal passband insertion loss and stopband rejection. The effects of the additional LC resonators on passband insertion loss and stopband rejection are analyzed and discussed. Finally, a $0.61 \times 0.39 \mathrm{~mm}^{2}$ chip filter is designed and fabricated on a GaAs substrate with nitride dielectric layers, the minimum insertion loss is only $1.5 \mathrm{~dB}$, the passband is 3.1-9.0GHz, the maximum return loss is lower than $-11 \mathrm{~dB}$, the rejection is up to $-34 \mathrm{~dB}$ at $2 \mathrm{GHz}$ and $-33 \mathrm{~dB}$ at $12 \mathrm{GHz}$, and the group delay variation is only $0.27 \mathrm{~ns}$ during the whole passband. These results demonstrated that the proposed filter can be considered as one of the most compact UWB filters with low passband loss and high stopband rejection. 
Author Contributions: Conceptualization, S.H. and B.Z.; Data curation, Z.C., S.H., and Y.H.; Formal analysis, Y.G. (Yiting Gao) and X.Z. (Xiaodong Zhang); Investigation, Y.G. (Yiting Gao), X.Z. (Xinlei Zhang), and Y.H.; Methodology, S.H. and B.Z.; Writing-original draft, S.H. and Z.C.; Writing-review and editing, Z.W., Z.C., and Y.G. (Yufeng Guo). All authors have read and agreed to the published version of the manuscript.

Funding: This work was financially supported by the National Natural Science Foundation of China (61501261), and the China Postdoctoral Science Foundation (2017M621793).

Conflicts of Interest: The authors declare no conflict of interest.

\section{References}

1. Hao, Z.C.; Hong, J.S. Ultrawideband filter technologies. Microw. Mag. IEEE 2010, 11, 56-68. [CrossRef]

2. Yassin, A.; Nasser, Y.; Awad, M.; Al-Dubai, A.; Raulefs, R. Recent advances in indoor localization: A survey on theoretical approaches and applications. IEEE Commun. Surv. Tutor. 2017, 19, 1327-1346. [CrossRef]

3. Wei, F.; Wu, Q.Y.; Shi, X.W.; Chen, L. Compact UWB bandpass filter with dual notched bands based on SCRLH resonator. IEEE Microw. Wirel. Compon. Lett. 2011, 21, 28-30. [CrossRef]

4. Sekar, V.; Entesari, K. Miniaturized UWB bandpass filters with notch using slow-wave CPW multiple-mode resonators. IEEE Microw. Wirel. Compon. Lett. 2011, 21, 80-82. [CrossRef]

5. Xu, J.; Wu, W.; Kang, W.; Miao, C. Compact UWB bandpass filter with a notched band using radial stub loaded resonator. IEEE Microw. Wirel. Compon. Lett. 2012, 22, 351-353. [CrossRef]

6. Sarkar, P.; Ghatak, R.; Pal, M.; Poddar, D.R. Compact UWB bandpass filter with dual notch bands using open circuited stubs. IEEE Microw. Wirel. Compon. Lett. 2012, 22, 453-455. [CrossRef]

7. Shen, Y.; Law, C.L. Compact UWB bandpass filter with high suppression at 2.4 and 5.8 ghz. Electron. Lett. 2012, 48, 851-853. [CrossRef]

8. Wu, Z.; Shim, Y.; Rais-Zadeh, M. Miniaturized UWB filters integrated with tunable notch filters using a silicon-based integrated passive device technology. IEEE Trans. Microw. Theory Tech. 2012, 60, 518-527. [CrossRef]

9. Zhang, X.Y.; Zhang, Y.W.; Xue, Q. Compact band-notched UWB filter using parallel resonators with a dielectric overlay. IEEE Microw. Wirel. Compon. Lett. 2013, 23, 252-254. [CrossRef]

10. Zhu, H.; Chu, Q.X. Compact ultra-wideband (UWB) bandpass filter using dual-stub-loaded resonator (DSLR). IEEE Microw. Wirel. Compon. Lett. 2013, 23, 527-529. [CrossRef]

11. Li, X.; Ji, X. Novel compact UWB bandpass filters design with cross-coupling between lambda/4 short-circuited stubs. IEEE Microw. Wirel. Compon. Lett. 2014, 24, 23-25. [CrossRef]

12. Sarkar, P.; Ghatak, R.; Pal, M.; Poddar, D.R. High-selective compact UWB bandpass filter with dual notch bands. IEEE Microw. Wirel. Compon. Lett. 2014, 24, 448-450. [CrossRef]

13. Kumar, S.; Gupta, R.D.; Parihar, M.S. Multiple band notched filter using c-shaped and e-shaped resonator for UWB applications. IEEE Microw. Wirel. Compon. Lett. 2016, 26, 340-342. [CrossRef]

14. Kamma, A.; Das, R.; Bhatt, D.; Mukherjee, J. Multi-mode resonators based triple band notch UWB filter. IEEE Microw. Wirel. Compon. Lett. 2017, 27, 120-122. [CrossRef]

15. Zhou, C.X.; Guo, P.P.; Zhou, K.; Wu, W. Design of a compact UWB filter with high selectivity and superwide stopband. IEEE Microw. Wirel. Compon. Lett. 2017, 27, 636-638. [CrossRef]

16. Huang, C.C.; Fang, W.T.; Lin, Y.S. Miniaturization of broadband stub bandpass filter using bridged-t coils. IEEE Access 2018, 6, 20164-20173. [CrossRef]

17. Xinlin, X.; Xu, C.; Fengjun, C.; Xianjin, D. Compact UWB bandpass filter with sharp roll-off using APCL structure. Electron. Lett. 2018, 54, 837-839.

18. Xuemei, Z.; Yuwen, P.; Tao, J. UWB bandpass filter with dual notched bands using t-shaped resonator and 1-shaped defected microstrip structure. Micromachines 2018, 9, 280-291.

19. Lei, H.; Naichang, Y. A Compact Wideband SIW Bandpass Filter with Wide Stopband and High Selectivity. Electronics 2019, 8, 440.

20. Badiaa, A.A.; Hicham, K.; Otman, A.; Ana, V.A. Selectivity and Rejection Bandwidth Improvements of a Multi-Band Stopband Filter Based on Circular Split Ring Resonator. In Proceedings of the 13th European Conference on Antennas and Propagation, Małopolskie, Poland, 1-5 April 2019.

21. Cao, H.; Ying, W.; Li, H.; Yang, S. Compact lowpass filter with wide stopband using novel windmill resonator. J. Electromagn. Waves Appl. 2012, 26, 2234-2241. [CrossRef] 
22. Naghshvarian, J.M.; Tayarani, M. Defected ground structure band-stop filter by semicomplementary split ring resonators. IET Microw. Antennas Propag. 2011, 5, 1386-1391. [CrossRef]

23. Liu, K.; Frye, R.C.; Emigh, R. Miniaturized ultra-wideband band-pass-filter from silicon integrated passive device technology. In Proceedings of the IEEE International Microwave Symposium Digest, Boston, MA, USA, 7-12 June 2009.

24. Lu, M.C.; Chang, J.F.; Lin, Y.S.; Lu, L.C. 1.89 dB insertion-loss UWB bp-filter with three finite transmission zeros using standard $0.18 \mu \mathrm{m}$ CMOS technology. Electron. Lett. 2009, 45, 56-57. [CrossRef]

25. Dine, W.G.E.; Ezzeddine, H.; Bila, S.; Verdeyme, S. Three approaches for the realization of a Chebyshev cross-coupled UWB filter. In Proceedings of the IEEE Microwave Symposium Digest, Anaheim, CA, USA, 23-28 May 2010.

26. Manchec, A.; Laporte, C.; Cédric, Q.; Ezzeddine, H.; Clavet, Y.; Rius, E. Hybrid lumped/distributed band-pass filter in IPD technology for ultra-wideband applications. In Proceedings of the IEEE Microwave Symposium Digest, Baltimore, MD, USA, 5-10 June 2011.

27. Tseng, T.H.; Fang, W.T.; Lin, Y.S. Super compact and ultra-wideband bandpass filter using bridged-T coils. In Proceedings of the IEEE Microwave Symposium Digest, San Francisco, CA, USA, 22-27 May 2016.

(C) 2020 by the authors. Licensee MDPI, Basel, Switzerland. This article is an open access article distributed under the terms and conditions of the Creative Commons Attribution (CC BY) license (http://creativecommons.org/licenses/by/4.0/). 